\title{
Remoción de Arsénico (V) en Solución Acuosa por Biomasa Modificada del Hongo Aspergillus niger
}

\author{
Erik E. Santos-Domínguez ${ }^{(1)}$, Juan M. Vargas-Morales ${ }^{(1)}$, Juan F. Cárdenas-González ${ }^{(2)}$, e Ismael \\ Acosta-Rodríguez ${ }^{(1)}$ \\ (1)Universidad Autónoma de San Luis Potosí, Facultad de Ciencias Químicas, Centro de Investigación y \\ de Estudios de Posgrado, Laboratorio de Micología Experimental. Av. Dr. Manuel Nava No. 6, \\ Zona Universitaria, 78320 San Luis Potosí, S.L.P. México. \\ (2)Universidad Autónoma de San Luis Potosí, Unidad Académica Multidisciplinaria Zona Media. Carretera \\ Río Verde san Ciro Km 4.5. Colonia Puente del Carmen, 79617 Río Verde San Luis Potosí, México. \\ (e-mail: iacosta@uaslp.mx).
}

Recibido Abr. 13, 2017; Aceptado Jun. 19, 2017; Versión final Ago. 9, 2017, Publicado Dic. 2017

\begin{abstract}
Resumen
Se analizó la capacidad de remoción de arsénico (As V) en solución acuosa por la biomasa modificada del hongo Aspergillus niger, por espectrometría de absorción atómica. La contaminación por arsénico es muy grave en México, afecta a casi todas las poblaciones, y las técnicas de remoción son muy caras y no tan eficientes. En los estudios, la mayor bioadsorción (69\% con $1 \mathrm{mg} \mathrm{L}^{-1}$ del metaloide) fue a las 24 horas, a $\mathrm{pH}$ de $6.0,28^{\circ} \mathrm{C}$ y $1 \mathrm{~g}$ de biomasa modificada. Con respecto a la temperatura, la más alta remoción fue a los $28^{\circ} \mathrm{C}$, con un $69 \%$ de remoción a las 24 horas. A mayor concentración de arsénico fue menor la remoción, y al aumentar la concentración del bioadsorbente, la remoción es más eficiente ( $96 \%$ con $5 \mathrm{~g}$ de biomasa). Además se remueve eficientemente el metaloide in situ (56\% de remoción en agua natural

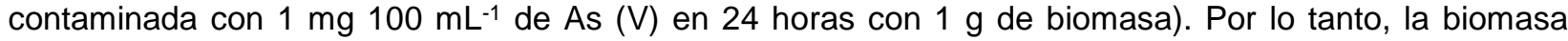
modificada se puede utilizar para eliminar el arsénico de aguas residuales industriales.
\end{abstract}

\section{Removal of Arsenic /V) in Aqueous Solution by Modified Fungal Biomass of Aspergillus niger}

The removal capacity of arsenic (As $\mathrm{V}$ ) in aqueous solution by the modified fungal biomass of Aspergillus niger, by atomic absorption spectrometry, was analyzed. Arsenic contamination is very serious in Mexico, it affects almost all populations, and the removal techniques are very expensive and not very efficient. In this study, the highest bioadsorption (69\% with $1 \mathrm{mg} \mathrm{L}^{-1}$ of the metalloid) was at 24 hours at $\mathrm{pH} 6.0,28^{\circ} \mathrm{C}$ and 1 $\mathrm{g}$ of modified biomass. With respect to the temperature, the highest removal was at $28^{\circ} \mathrm{C}$, with a $69 \%$ removal in 24 hours. At higher concentration of arsenic the removal was lower, and by increasing the concentration of the bioadsorbent, the removal is more efficient ( $96 \%$ with $5 \mathrm{~g}$ of biomass). Additionally, the biomass efficiently removes the metalloid in situ (56\% removal in natural water contaminated with $1 \mathrm{mg} 100$ $\mathrm{mL}^{-1}$ of As (V) in 24 hours with $1 \mathrm{~g}$ of biomass). Therefore, the modified biomass can be used to eliminate arsenic from industrial wastewater.

Keywords: bioadsorption; arsenic; cellular fungal biomass modified; bioremediation 
Remoción de Arsénico (V) en Solución Acuosa por Biomasa Modificada del Hongo Santos-Domínguez

\section{INTRODUCCIÓN}

La contaminación del medio ambiente por productos derivados del petróleo, compuestos farmacéuticos, cloro, nitrofenoles y sus derivados, hidrocarburos aromáticos policíclicos, tintes orgánicos, plaguicidas y metales pesados es un problema muy serio, con grandes consecuencias en la salud de los organismos vivos, la falta de agua, alimentos e insumos aptos para el consumo humano (Dzionek et al., 2016). Los efectos tóxicos del arsénico afectan a personas de todas las edades, principalmente aquellas que viven en la pobreza y con desnutrición: niños, mujeres embarazadas y en lactancia, e individuos con enfermedades crónicas. Además, se ha relacionado su exposición crónica por el agua de consumo humano a cáncer de piel, vejiga, riñón y próstata (Basu et al., 2014). La Organización Mundial de la Salud recomienda una concentración máxima de arsénico de $10 \mu \mathrm{g} \mathrm{L}^{-1}$ como un valor de referencia para el agua potable (NOM-021-SEMARNAT-2000), y se estima que en América Latina por lo menos catorce millones de personas beben en forma permanente agua con niveles de arsénico que ponen en riesgo su salud (Bardach et al., 2015), debido a que las concentraciones de arsénico, sobre todo en el agua subterránea, presentan niveles que llegan en algunos casos hasta $1 \mathrm{mg}$ $\mathrm{L}^{-1}$ (Conapris, 2006). Las formas predominantes en tierra y agua son arsenito (As (III)) y arsenato (As (V)) (Yamani et al., 2012). El arsénico trivalente incluye las especies $\mathrm{As}(\mathrm{OH})_{3}, \mathrm{As}(\mathrm{OH})_{4}^{4}, \mathrm{AsO}_{2} \mathrm{OH}_{2}{ }^{-}$y $\mathrm{AsO}_{3}{ }^{-}$, mientras que el arsénico pentavalente incluye $\mathrm{AsO}_{4}{ }^{3-}, \mathrm{HAsO}_{4}{ }^{2-} \mathrm{y} \mathrm{H}_{2} \mathrm{AsO}^{4}$. Además, el As (III) es mucho más tóxico y móvil que el As (V) (Sahmoune, 2016), y usualmente se encuentra en aguas subterráneas y en aguas hidro-termales, mientras que el As (V) es común en aguas superficiales y aguas subterráneas oxidadas (aquellas que contienen compuestos que oxidan el As (III) a As (V, como materia orgánica de origen biológico).

En México, la exposición crónica al arsénico del agua subterránea, se describió inicialmente en 1958 como un problema endémico en la Comarca Lagunera (gran parte de los estados de Durango y Coahuila) (Jasso Pineda et. al., 2017). En 1962, se reportaron 40 casos severos y una muerte, en el área urbana de Torreón (estado de Coahuila). Posteriormente, se encontró que la presencia del metaloide en agua potable proveniente de fuentes de agua subterránea, era un problema en muchos otros estados de la República Mexicana, como Baja California, Durango, Coahuila, Zacatecas, Morelos, Aguascalientes, Chihuahua, Puebla, Nuevo León, Guanajuato, Jalisco, Oaxaca y San Luis Potosí; donde se encontraron concentraciones de arsénico que excedían los valores nacionales e internacionales regulatorios en el agua potable (10-50 $\mu \mathrm{g}$ $\mathrm{L}^{-1}$ ) (Ramírez Meda et. al., 2017; WHO 2011), y una gran contaminación en el acuífero colgado del Valle de San Luis Potosí (López Alvarez et. al., 2013).

En el estado de San Luis Potosí, el agua subterránea es la principal fuente de abastecimiento en la zona centro y altiplano del estado, los acuíferos se encuentran a profundidades cada vez mayores, con el subsecuente aumento de contaminantes inorgánicos y metales pesados. Desde el año 2003, de forma periódica los servicios de salud del estado realizan la evaluación de la calidad del agua en fuentes de abastecimiento para evidenciar la presencia de algunos parámetros que rebasen la normatividad correspondiente (NOM-127-SSA1-1994). Se ha encontrado arsénico en algunas localidades de los municipios de Ahualulco, Villa de Ramos, Salinas, Guadalcazar, Mexquitic de Carmona y la capital del estado. En Villa de Ramos, se han reportado concentraciones de arsénico de hasta $0.179 \mathrm{mg} \mathrm{L}^{-1}$. Dentro del mismo, se encuentran tres localidades con concentraciones por arriba del valor de la Norma Oficial Mexicana. En Salinas, en la localidad La Reforma, se presentan concentraciones de $0.056 \mathrm{mg} \mathrm{L}^{-1}$ y en Santo Domingo, la localidad de San Juan del Salado presenta un valor de $0.0355 \mathrm{mg} \mathrm{L}^{-1}$, las cuales rebasan la concentración de arsénico establecida por la Norma de 0.0025 $\mathrm{mg} \mathrm{L}^{-1}$, en la modificación a la NOM-127-SSA1-1994 (Coronado et al., 2012). Por otro lado, en el distrito minero de San Luis de la Paz, en Villa de la Paz (8 km de la ciudad de Matehuala), se han reportado $158 \mathrm{mg} \mathrm{L}^{-1}$ de arsénico disuelto, y otras concentraciones en el agua de riego de suelos agrícolas y cultivos de maíz (MartínezVillegas et al., 2013; Ruíz Huerta et. al., 2017), además, las aguas en contacto con los sedimentos, presentan concentraciones de arsénico de hasta $200,000 \mu \mathrm{m} \mathrm{L}^{-1}$, y los sedimentos analizados presentan concentraciones totales de arsénico de 143.00 a $2054.60 \mathrm{mg} \mathrm{Kg}^{-1}$ (Torres y Martínez, 2010): también, se ha evaluado el riesgo sanitario de algunos sitios mineros contaminados con arsénico y plomo en Villa de la Paz (Jasso Pineda et. al., 2017), y se ha reportado una gran contaminación en el acuífero colgado del Valle de San Luis Potosí por diferentes fuentes (López Alvarez et. al., 2013).

Por otro lado, se ha tratado de eliminar el arsénico de nichos contaminados, con algunos métodos como: coagulación-floculación, precipitación, adsorción, intercambio iónico y filtración en membrana, pero la mayoría son muy caros y no hay una remoción total, tienen baja selectividad y presentan dificultades para la eliminación de lodos tóxicos (Mathew et. al., 2016). También, se ha estudiado la remediación en agua para consumo humano con resinas de intercambio iónico y por hongos aislados de suelos con resultados positivos (Prieto-García et. al., 2012; Srivastava et. al., 2011). Se ha observado un aumento en la remoción de As (V) por huesos carbonizados de animales cubiertos con manganeso (Liu et. al., 2016), la biomasa modificada de diferentes hongos (Cárdenas-González et. al., 2015), así como otras biomasas biológicas (Srivastava y Dwivedi, 2015). El objetivo de este trabajo fue analizar la capacidad de remoción de As (V) en solución acuosa por la biomasa modificada de Aspergillus niger. 


\section{METODOLOGÍA}

Se trabajó con una cepa de $A$. niger que crece en $300 \mathrm{ppm}$ de As (V), y que fue aislada a partir del aire contaminado de una zona aledaña a la Facultad de Ciencias Químicas de la UASLP (en una gasolinera, a un lado de una glorieta que tiene una gran circulación vehicular), San Luis Potosí, S.L.P., México. La cepa fúngica se mantuvo de manera rutinaria en agar papa dextrosa. Para la obtención de la biomasa, se sembraron $1 \mathrm{x}$ $10^{6}$ esporas $\mathrm{mL}^{-1}$ del hongo, en matraces Erlenmeyer de $1 \mathrm{~L}$, conteniendo $500 \mathrm{~mL}$ de caldo Sabouraud dextrosa, incubando durante 5 días a $28^{\circ} \mathrm{C}$ y $100 \mathrm{rpm}$. Posteriormente, se obtuvo la biomasa por filtración, se lavó con $200 \mathrm{~mL}$ de agua tridesionizada y se colocó en cajas de Petri incubando a $70^{\circ} \mathrm{C}$ en un horno hasta sequedad, después se pulverizó en un mortero, y se guardó en un frasco ámbar estéril hasta su uso. Para la preparación de la biomasa cubierta con óxido de hierro, se tomaron $20 \mathrm{~g}$ de la biomasa fúngica, y se le añadieron $80 \mathrm{~mL}$ de $\mathrm{Fe}\left(\mathrm{NO}_{3}\right)_{3} \cdot 9 \mathrm{H}_{2} \mathrm{O} 2 \mathrm{M}$ y $1 \mathrm{~mL}$ de $\mathrm{NaOH} 10 \mathrm{M}$, se homogenizo la solución en un recipiente de porcelana (crisol), por agitación a $100 \mathrm{rpm}$ por 15 minutos, y se incubó durante $3 \mathrm{~h} \mathrm{a} 80^{\circ} \mathrm{C}$, y posteriormente a $120^{\circ} \mathrm{C}$ durante $24 \mathrm{~h}$. Se enfrió 1 hora a temperatura ambiente, y la biomasa tratada se homogenizó en un mortero (Pokhrel y Viraraghavan, 2006).

Se trabajó con $100 \mathrm{~mL}$ de una solución de $1 \mathrm{mgL}^{-1}$ de As (V) obtenida por dilución de una solución patrón de $100 \mathrm{mgL}^{-1}$ a partir de $\mathrm{Na}_{2} \mathrm{HAsO}_{4}$ en agua tridesionizada. Se ajustó el pH de la solución a analizar con $\mathrm{HNO}_{3} 1$ $\mathrm{M}$ y/o NaOH $1 \mathrm{M}$, antes de adicionarla a la biomasa modificada. Posteriormente, se hicieron diluciones a concentraciones de 1 a $5 \mathrm{mgL}^{-1}$ del metaloide. Para los estudios de remoción, a matraces Erlenmeyer de 250 $\mathrm{mL}$ que contenían $1.0 \mathrm{~g}$ de la biomasa modificada (previamente esterilizada a $120^{\circ} \mathrm{C} / 20$ minutos), se les adicionaron $100 \mathrm{~mL}$ de una solución de $1 \mathrm{mg} \mathrm{L}^{-1}$ de As $(\mathrm{V})$ incubando a $28^{\circ} \mathrm{C}$ y $100 \mathrm{rpm}$, tomando alícuotas de $5 \mathrm{~mL}$ a $0,4,8,12,16,20,24$ y 28 horas, las cuales se centrifugaron a $3000 \mathrm{rpm}$ (5 min), y al sobrenadante respectivo se le determinó la concentración de iones arsénico en solución por Espectrometría de Absorción Atómica por generación de Hidruros (Espectrómetro de Absorción Atómica Varian, modelo Spectra AA- 20), de acuerdo al procedimiento señalado por la Norma Oficial Mexicana (SSA, 1994). Todos los experimentos se realizaron un mínimo de 3 veces y por triplicado. También, se realizó un estudio de biorremediación en $100 \mathrm{~mL}$ agua natural contaminada con $1 \mathrm{mg} \mathrm{L}^{-1}$ de As (ajustado), colectada en Cerrito Blanco, Matehuala, San Luis Potosí, México, se ajustó el $\mathrm{pH}$ a 6.0 , y se agregó $1.0 \mathrm{~g}$ de biomasa fúngica modificada, incubando a $28^{\circ} \mathrm{C}$ con agitación constante durante 24 h, tomando muestras a las 0 y 24 h, y se determinó la concentración de iones As (V) en solución.

Se realizó un estudio con enfoque cuantitativo, analítico, explicativo y experimental. La información obtenida fue procesada y analizada mediante el programa SPSS v.20 (SPSS Inc., Chicago Illinois) y Microsoft Office Excel Professional Plus 2010, formato .xlsx. Se emplearon tablas y figuras para la presentación descriptiva de los resultados. Así mismo para encontrar asociación entre las variables se realizó la prueba estadística t de student, con una significancia estadística $<0.05$.

\section{RESULTADOS Y DISCUSIÓN}

En la Figura 1, se observa el efecto de la biomasa nativa y modificada de $A$. niger, y del pH sobre la remoción de As $(V)$ en solución, encontrando que la remoción con la biomasa natural es de $10.1 \pm 0.15 \%$ (IC 95\% 9.710.5) y con un $\mathrm{pH} \neq 6$ de $5.3 \pm 2.4 \%$, (IC $95 \% 4.0-6.4)$ con una diferencia significativa ( $\mathrm{p}=0.003$ ).

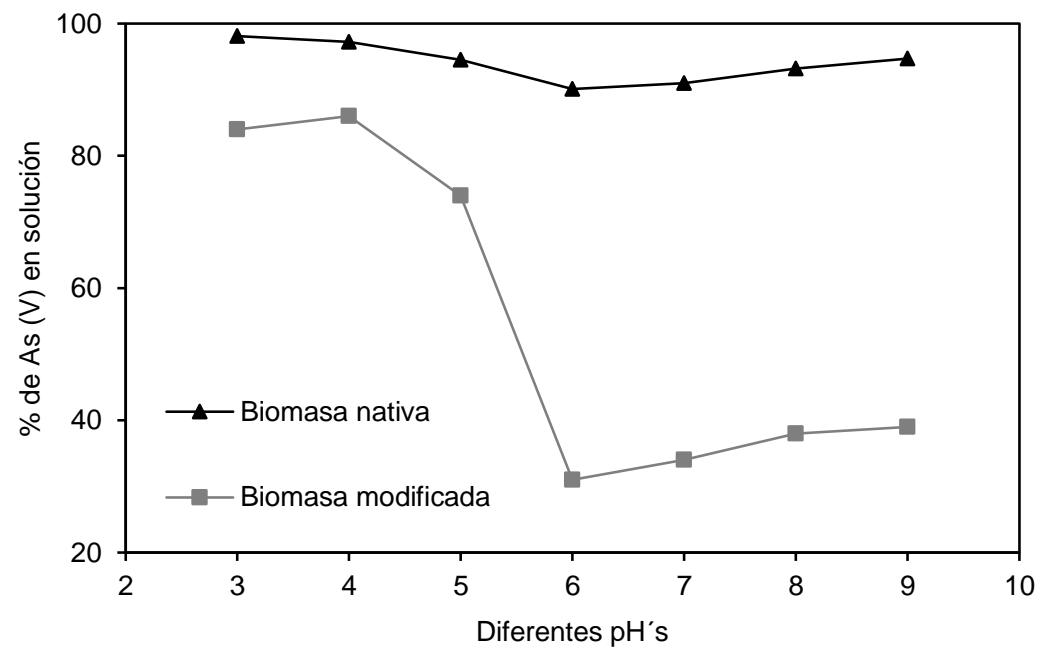

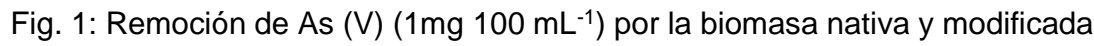
de $A$. niger $(1 \mathrm{~g}) .28^{\circ} \mathrm{C}$. 24 h. $100 \mathrm{rpm}$ a diferentes $\mathrm{pH}$ 's. 
Estos resultados son similares a los reportados para la biomasa natural de Paecilomyces sp (10.1\%) (CárdenasGonzález et. al., 2015), los huesos carbonizados de animales cubiertos con manganeso (1.2\%) (Liu et. al., 2016), para materiales derivados de Maracuyá (Passiflora edulis) (5.95\%) (llina et. al. 2009), y para la biomasa modificada de A. niger para la remoción de As (III) Santos et al., 2017). Se ha reportado que las propiedades estructurales del bioadsorbente, como el soporte celular y otros factores, afectan la capacidad de remoción del As (III) (Sahmoune, 2016). Con respecto a la biomasa tratada con óxido de hierro, la remoción fue muy eficiente (69\%, pH 6.0, 24 h de incubación) (Figura 1). El metaloide, es removido más eficientemente a un pH de 6.0, debido a que a este $\mathrm{pH}$ es parcialmente ionizado a la forma $\mathrm{H}_{2} \mathrm{AsO}^{-3} \mathrm{y}$ forma complejos con la biomasa tratada, mientras que a pH's mayores, se incrementan en la solución los iones $\mathrm{OH}^{-}$, y compiten con los iones de As (V) en la solución, y por lo tanto la remoción es menos eficiente (Yamani et al., 2012), con una diferencia ( $\mathrm{p}=0.001$ ), a un $\mathrm{pH}$ de $6(89 \pm 1 \%$, IC $95 \% 88-90)$ y con un $\mathrm{pH} \neq 6(41.2 \pm 21.2 \%$, IC $95 \% 32.4-51.5)$. Estos resultados son similares a otros reportes como: Paecilomyces sp., y A. niger tratados de manera similar, para la remoción de As (V) y As (III) (Cárdenas-González et. al., 2015; Santos et al., 2017), el micelio modificado de Aspergillus fumigatus (Sathishkumar et. al., 2004), materiales derivados de Maracuyá tratados con Fe (III) (llina et. al. 2009) y la biomasa de $A$. niger cubierta con óxido de hierro (Pokhrel y Viraraghavan, 2006).

En la Figura 2, se observa la remoción de As $(\mathrm{V})$ por la biomasa fúngica modificada a diferentes tiempos de incubación y pH de 6.0, encontrando que a mayor tiempo de incubación es mayor la remoción $(69 \%$ a las 28 h), lo cual indica mayor disponibilidad de los sitios de unión del bioadsorbente (Raje y Swain, 2002), con una diferencia ( $\mathrm{p}=0.022)$ en la media, con 24 horas de incubación $(89 \pm 1 \%$, IC $95 \% 88-90)$ y con menos de 24 horas de incubación (48.4 $\pm 27.6 \%$, IC95\% 35.8-60.9).

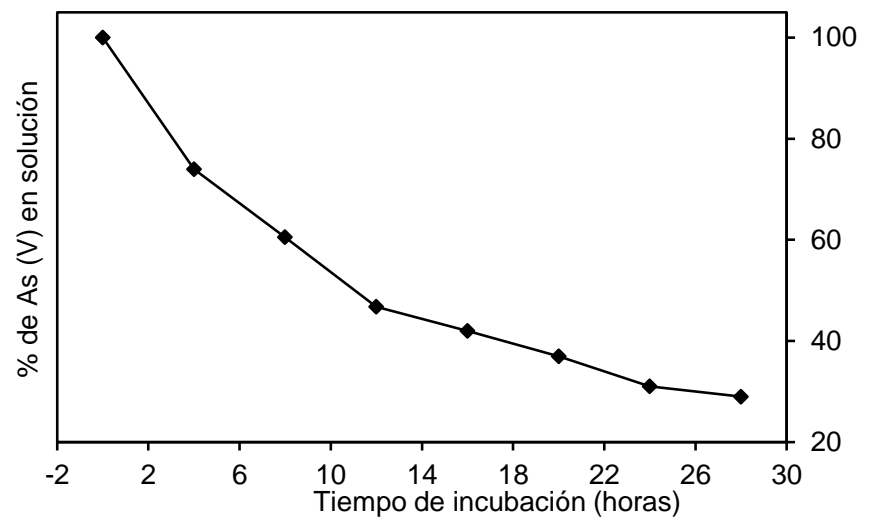

Fig. 2: Efecto del tiempo de incubación sobre la remoción de $1 \mathrm{mg} 100$ $\mathrm{mL}^{-1}$ de As $(\mathrm{V}) .28^{\circ} \mathrm{C} .100 \mathrm{rpm}$. $1 \mathrm{~g}$ biomasa modificada. $\mathrm{pH} 6.0$.

Después de este tiempo, no hay un aumento significativo en el porcentaje de remoción del metaloide. Estos resultados son similares a los reportados para la biomasa del hongo macromiceto Trametes versicolor modificada con polietilenimina (Song et. al., 2015), para los hongos Paecilomyces sp. y A. niger tratados de manera similar para la remoción de As (V) y As (III) (Cárdenas-González et. al., 2015; Santos et al., 2017), la oxidación de As (V) por hidróxidos estructurales de Fe (II) Shao et. al., 2016) y para el alga Botryococcus braunii (Podder y Majumder, 2016). Por otra parte, la capacidad de remoción es mejor a menor temperatura ( $p=0.001$ ) (Figura 3), con una media de $89 \pm 1 \%$ (IC 95\% 88-90) y a más de $30^{\circ} \mathrm{C}$ de $79.2 \pm 3.3 \%$ (IC $95 \%, 77.1$ $81.2)$, con una diferencia significativa $(p=0.001)$.

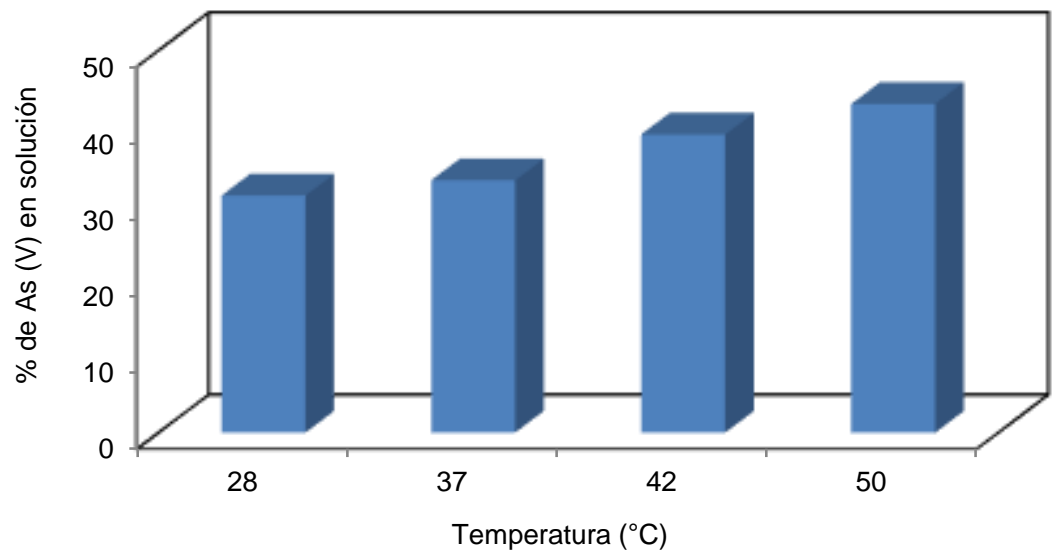

Fig. 3: Efecto de la temperatura de incubación sobre la remoción de $1 \mathrm{mg} 100 \mathrm{~mL}^{-1} \mathrm{de}$ As (V) por $1 \mathrm{~g}$ de biomasa modificada de $A$. niger. 24 h. pH 6.0. 100 rpm. 
Lo cual es similar a lo reportado para las biomasas modificadas con óxido férrico de Paecilomyces sp. (Cárdenas-González et. al., 2015), materiales derivados de Maracuyá ( $P$. edulis) tratados con Fe (III) (Ilina et. al. 2009) y la biomasa de $A$. niger cubierta con óxido de hierro (Pokhrel y Viraraghavan, 2006), Pero, son diferentes a los reportados para la biomasa del hongo macromiceto $T$. versicolor modificada con polietilenimina (Song et. al., 2015) y para la biomasa de la cáscara de Citrus limetta (Kamsonlian et. al., 2013). La temperatura puede ser importante para los mecanismos dependientes de energía en la bioadsorción de metales por microorganismos, aunque la bioadsorción de As (V) por el hongo A. niger, parece ser independiente de la temperatura analizada.

Con respecto al efecto de diferentes concentraciones de As $(\mathrm{V})$ en solución (1-5 $\left.\mathrm{mg} \mathrm{L}^{-1}\right)$, sobre la remoción del mismo, se encontró que, a mayor concentración, disminuye su remoción pues con $1 \mathrm{mg} \mathrm{L}^{-1}$ de As (V) se remueve el $69 \%$, mientras que con $5 \mathrm{mg} \mathrm{L}^{-1}$ se elimina un $48 \%$ a las $24 \mathrm{~h}$ (Figura 4), lo cual probablemente se debe a que el número de iones adsorbido de la solución a bajas concentraciones, es mayor que los adsorbidos a partir de soluciones con altas concentraciones del metaloide.

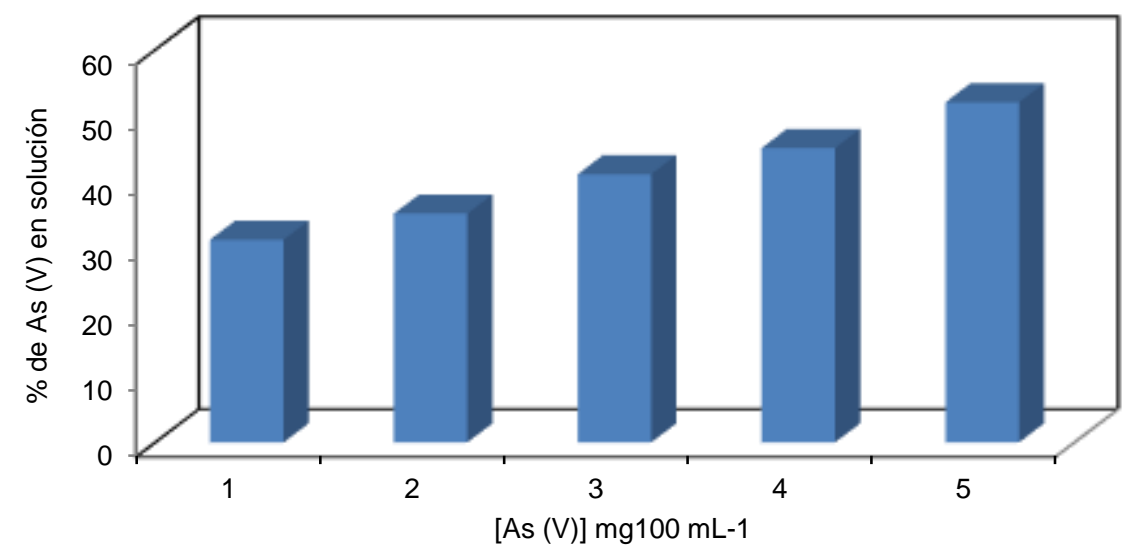

Fig. 4: Efecto de la $[\mathrm{As}(\mathrm{V})]$ en solución sobre la remoción del mismo. pH 6.0.1.0 g biomasa modificada. $28^{\circ} \mathrm{C} .24 \mathrm{~h}$ de incubación.

Resultados similares se han reportado para el micelio modificado de A. fumigatus (Sathishkumar et. al., 2004), la biomasa modificada con óxido férrico de Paecilomyces sp., (Cárdenas-González et. al., 2015), el alga $B$. braunii (Podder y Majumder, 2016), diferentes especies de Bacillus y Lysinibacillus (Eman et. al., 2015) y para las bacterias Bacillus macerans, Bacillus megaterimand y Corynebacterium vitarumen, aisladas de suelos contaminados (Ghodis et. al., 2011).

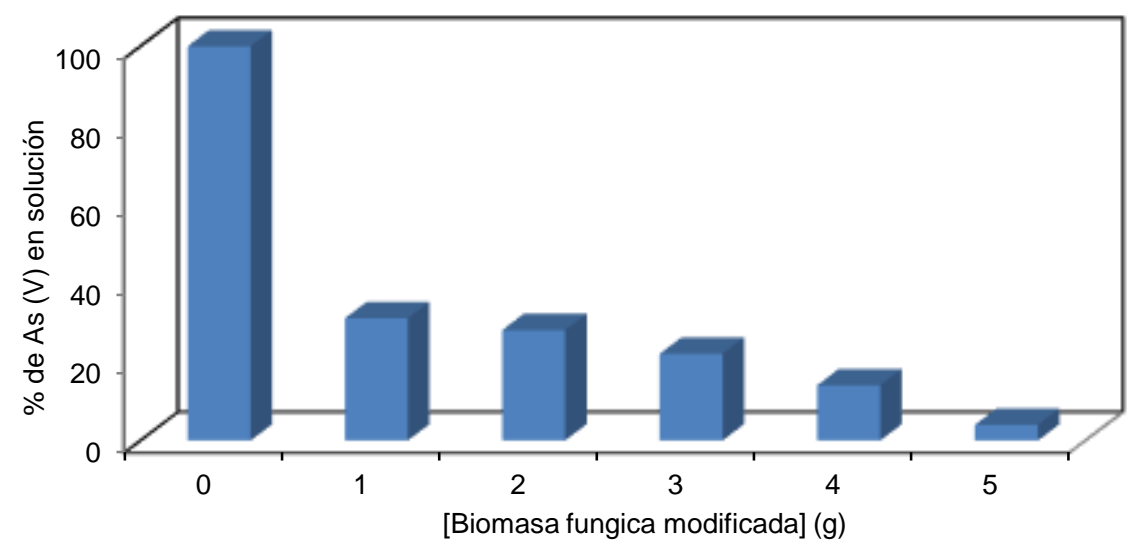

Fig. 5: Efecto de la [biomasa fúngica modificada] sobre la remoción de $1 \mathrm{mg} 100 \mathrm{~mL}^{-1}$ de As (V). 100 rpm. PH $6.0 .28^{\circ} \mathrm{C} .24 \mathrm{~h}$ de incubación.

Por otro lado, a mayor concentración de la biomasa modificada, hay mayor remoción del metaloide en solución (96\% de remoción con $5 \mathrm{~g}$, a las 24 h) (Figura 5), lo cual se puede deber a que hay más sitios de bioadsorción del metal, pues la cantidad de bioadsorbente añadido determina el número de sitios de unión disponibles para la bioadsorción del metal (Tuzen et al., 2009). Resultados similares se han reportado para la biomasa de la cáscara de C. limetta (Kamsonlian et. al., 2013), el alga B. braunii (Podder y Majumder, 2016) y la biomasa modificada con óxido férrico de Paecilomyces sp. (Cárdenas-González et. al., 2015). Además, analizando la

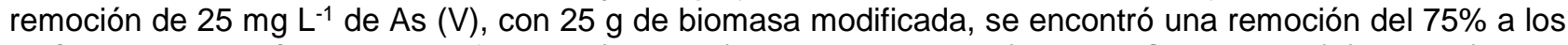
7 días de incubación, con una diferencia $(\mathrm{p}=0.002)$ entre la media de $(75 \pm 3 \%$, IC95\% 72-78) (Figura 6). 


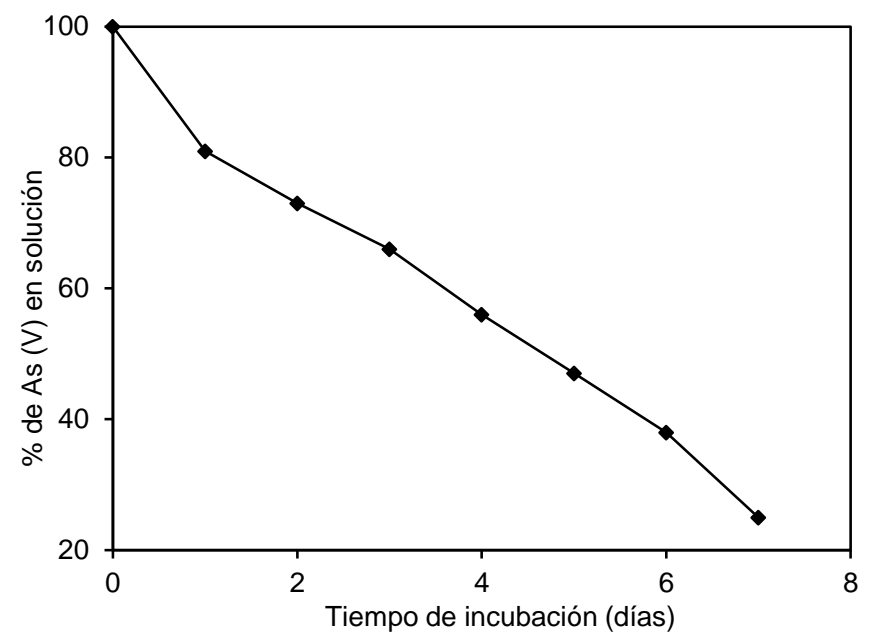

Fig. 6: Remoción de As (V) por $10 \mathrm{~g}$ de biomasa modificada de A. niger. $25 \mathrm{mg} \mathrm{L}^{-1}$ de As (V). 100 rpm. $28^{\circ} \mathrm{C}$. pH 6.0 .

Finalmente, la biomasa fúngica modificada, se utilizó para la remoción de As (V) de muestras de agua contaminadas con $1 \mathrm{mg} \mathrm{L}^{-1} \mathrm{~L}$, procedentes de Zimapan, estado de Hidalgo, México, sitio en el cual, el agua subterránea está altamente contaminada de manera natural con As (0.6 mg L-1) (Prieto-García et. al., 2012), lo cual indica que esta concentración es mayor al límite máximo permitido para el agua de beber $\left(0.5 \mathrm{mg} \mathrm{L}^{-1}\right)$ de acuerdo a la NOM-127-SSA1-1994, y de $0.25 \mathrm{mg} \mathrm{L}^{-1}$ de acuerdo a la NOM-127-SSA1-1994-2000 (1994). Los resultados obtenidos se muestran en la Tabla 1, donde se observa que después de $24 \mathrm{~h}$ de incubación, la concentración de As (V) se reduce a un valor de $0.44 \mathrm{mg} \mathrm{L}^{-1}$ (56\% de remoción), lo que indica la eficiencia del material bioadsorbente para la remoción del metaloide de las muestras de agua. Estos resultados son parecidos para la remoción de As (V) con la biomasa modificada con óxido férrico de Paecilomyces sp. (Acosta-Rodríguez et al., 2013), el alga B. braunii (Podder y Majumder, 2016), resinas de intercambio iónico (Prieto-García et al., 2012), arena cubierta con óxido de fierro (Thirunavukkarasu et. al., 2003) y materiales derivados de Maracuyá tratados con Fe (III) (Iliná et al., 2009), y para la remoción de As (V) por nueve biomasas fúngicas modificadas con óxido férrico (Cárdenas-González et al., 2017).

Tabla 1: Remoción de As (V) de agua natural contaminada con $1.0 \mathrm{mg} \mathrm{L}^{-1} .1 \mathrm{~g}$ de biomasa modificada, $100 \mathrm{rpm}, 28^{\circ} \mathrm{C}$, pH 6.0 (ajustado), 24 h de incubación

\begin{tabular}{|c|c|}
\hline Tiempo de Incubación(Horas) & \% de Remoción \\
\hline 0 & 0 \\
\hline 24 & 56 \\
\hline
\end{tabular}

\section{CONCLUSIONES}

La biomasa modificada de A. niger, remueve un $69 \%$ de As (V) en solución ( $\left.1 \mathrm{mg} \mathrm{L}^{-1}\right)$, a las 24 h de incubación, $28^{\circ} \mathrm{C}, 100 \mathrm{rpm}$ y $1 \mathrm{~g}$ de biomasa; y elimina eficientemente el metal in situ (56\% en agua contaminada con el metaloide). Estos resultados sugieren la potencial aplicabilidad de esta biomasa para la remediación de nichos acuáticos contaminados con As (V).

\section{REFERENCIAS}

Bardach, A.E., y otros 8 autores, Epidemiology of chronic disease related to arsenic in Argentina: A systematic review, Science of the Total Environment, 538, 802-816 (2015)

Basu, A., y otros 4 autores, A Review on Sources, Toxicity and Remediation Technologies Removing Arsenic from Drinking Water, Research on Chemical Intermediates, 40, 447-485 (2014)

Cárdenas-González, J.F., Terán-Figueroa, Y., Martínez-Juárez, V.M. y Acosta-Rodríguez, I., Removal of Arsenic (V) from Aqueous Solutions onto Fungal biomass, Jökull Journal, 65(6), 233-242 (2015)

Cárdenas-González, J.F., Acosta-Rodríguez, I., Terán Figueroa, Y. y Rodríguez-Pérez, A.S., Bioremoval of arsenic (V) from aqueous solutions by chemically modified biomass, 3 Biotech., 7(226), 1-6, Dio10.1007/s13205-0868-5 (2017) 
Conapris, Epidemiologia del Hidroarsenicismo crónico regional endémico HACRE en la República Argentina, estudio colaborativo multicéntrico. Ministerio de Salud de la Nación, 11-12, 18-19 (2006)

Coronado-Salas, C. y otros 6 autores, La comunicación de riesgos como una herramienta para disminuir la exposición infantil a plomo y arsénico en la zona contaminada de Villa de la Paz-Matehuala, San Luís Potosí, México, Revista Internacional de Contaminación Ambiental, 28(2), 167-181 (2012)

Dzionek, A., Wojcieszyńska, D. y Guzik, U., Natural carriers in bioremediation: A review, Electronic Journal of Biotechnology, 23, 28-36 (2016)

Eman, A., Mohamed, H. y Farag, A.G., Arsenic Removal from Aqueous Solutions by Different Bacillus and Lysinibacillus Species, Bioremediation Journal, 19(4), 269-276 (2015)

Ghodsi, H., Hoodai, M., Tahmourespour, A. y Gheisari, M.M., Investigation of bioremediation of arsenic by bacteria isolated from contaminated soil, African Journal of Microbiology Research, 5(32), 5889-5895 (2011)

Iliná, A., y otros 5 autores, Biosorción de arsénico en materiales derivados del Maracuyá, Revista Internacional de Contaminación Ambiental, 25(4), 201-216 (2009)

Jasso-Pineda, Y. y otros 10 autores, An Integrated Health Risk Assessment Approach to the Study of Mining Sites Contaminated With Arsenic and Lead, Integrated Environmental Assessment and Management 3(3), 344-350 (2017)

Kamsolian, S., Suresh, S., Majumder, C.B. y Chand, S., Biosorption of Arsenic by Mosambi (Citrus limetta) Peel: Equilibrium, Kinetics, Thermodynamics and Desorption Study, Asian J. of Chemistry, https://doi.org/10.14233/ajchem.2013.13336, 25(5), 2409-2417 (2013)

Litter, M.I., Taller de distribución de arsénico en Iberoamérica. Editorial Programa Iberoamericano de Ciencia y Tecnología para el Desarrollo, Buenos Aires, Argentina (2006)

López-Alvarez, B., y otros 4 autores, Origen de la calidad del agua del acuífero colgado y su relación con los cambios de uso de suelo en el Valle de San Luis Potosí. Boletín de la Sociedad Geológica Mexicana 65(1), 9-26 (2013)

Liu, J., He, L., Dong, F. y Hudson, K.A., The role of nano-sized manganese coatings on bone char in removing arsenic (V) from solution: implications for permeable reactive barrier technologies. Chemospere, 153, 146-154 (2016)

Martínez, N. y otros 7 autores, Arsenic mobility controlled by solid calcium arsenates: A case study in Mexico showcasing a potentially widespread environmental problem, Environmental Pollution, 176, 114-122 (2013)

Mathew, B.B., Jaishankar, M., Biju, V.G. y Beeregowda, K.N., Rol of biosorbentes in reducing toxic metals, Journal of Toxiclogy: Vol. 2016, Article ID 4369604, 13 pages. http://dx.doi.org/10.1155/2016/4369304 (2016)

Norma Oficial Mexicana: NOM-127-SSA1-1994. Salud ambiental, agua para uso y consumo humano-límites permisibles de calidad y tratamientos a que debe someterse el agua para su potabilización, México, 1-13 (1993)

Norma Oficial Mexicana: NOM 014-SSA1-1994. Sobre parámetros a determinar y la forma correcta de llevar el muestreo, conservación y manejo de las muestras hasta su ingreso al laboratorio, México, 97-105 (1993)

Poddeer, M.S. y Majumder, C.B., The use of artificial neural network for modelling of phycoremediation of toxic $\mathrm{As}(\mathrm{III})$ and $\mathrm{As}(\mathrm{V})$ from wastewater using Botryococcus braunii, Spectrochimica Acta Part A: Molecular and Biomolecular Spectroscopy, 155,130-145 (2016)

Pokhrel, D. y Viraraghavan, T., Arsenic removal from an aqueous solution by a modified fungal biomass, Water Research, 40, 549-552 (2006)

Prieto-García, F., Pérez, F. y Marmolejo, Y., Study of arsenic removal with ionic Exchange resins in drinking water from Zimapan, Hidalgo state, Mexico, Int. J. Applied Science and Technology, 2(6), 14-16 (2012)

Raje, N. y Swain, K.K., Purification of arsenic contaminated ground water using hydrated manganese dioxide, Journal of Radioanalytical and Nuclear Chemistry, 253 (1), 77-80 (2002) 
Ramírez-Meda, W., Bernal-Casillas, J.J., López-Tejeda, L.A. e Iñiguez-Covarrubias, G., Characteristics of toxic waste from nine casting industries according to Mexican Standards: Prospective study of West Mexico, Journal of Geoscience and Environment Protection, 5, 43-49 (2017)

Ruiz-Huerta, E.A. y otros 6 autores, Arsenic contamination in irrigation water, agricultural soil and maize crop from an abandoned smelter site in Matehuala México, Journal of hazardous Materials, in press (2017)

Sahmoune, M.N., The role of biosorbents in the removal of arsenic from water, Chemical Engineering and Technology, 39 (9), 1617-1628 (2016)

Santos, E. y otros 5 autores, Bioadsorción de arsénico (III) en solución acuosa por la biomasa modificada de Aspergillus niger, Avances en Ciencias e Ingeniería, 8 (2), 1-10 (2017)

Sathishkumar, M. y otros 5 autores, Bioremediation of arsenic contaminated groundwater by modified mycelial pellets of Aspergillus fumigatu, Bulletin of Environmental Contamination and Toxicology, 72, 617-624 (2004)

SEMARNAT. Norma Oficial Mexicana NOM-021-SEMARNAT-2000. "Establece las especificaciones de fertilidad, salinidad y clasificación de los suelos, estudio, muestreo y análisis". Secretaría del Medio Ambiente y Recursos Naturales. Diario Oficial de la Federación, México, 28-47 (2000)

Shao, B. y otros 4 autores, Advantages of aeration in arsenic removal and arsenite oxidation bystructural Fe(II) hydroxides in aqueous solution, Colloids and Surfaces A, 506, 703-710 (2016)

Song, W., Zhang, M., Liang, J. y Han, G., Removal of As (V) from wastewater by chemically modified biomass, Journal of Molecular Liquids, 206, 262-267 (2015)

Srivastava, P.J. y otros 5 autores, Biological removal of arsenic pollution by soli fungi, Science of the Total Environment, 409, 2430-2442 (2011)

Srivastava, S. y Dwivedi, A., Biological Wastes the Tool for Biosorption of Arsenic, Journal of Bioremediation and Biodegradation, 7(1), 1-3 (2015)

Thirunavukkarasu, O.S., Viraraghavan, T. y Subramanian, K.S., Arsenic removal from drinking water using iron oxide-coated sand, Water, Air, and Soil Pollution, 142, 95-111 (2003)

Torres-Reyes, A. y Martínez Villegas, N.V., Determinación de arsénico en sedimentos en el acuífero somero de Matehuala-Cerrito Blanco. 12ํㅡㄴ Verano de la Ciencia. UASLP. Edit. Universitaria Potosina, 1-9 (2010)

Tuzen, M. y otros 5 autores, Characterization of biosorption process of As(III) on green algae Ulothrix cylindricum, Journal of Hazardous Materials, 165(1-3), 566-572 (2009)

WHO, Guidelines for Drinking-water Quality. $4^{\text {th }}$ Ed. World Health Organization, Switzerland, https://goo.gl/RebK4G. 564 páginas, ISBN: 978924154815 1, 2011. Acceso: 14 de junio (2011)

Yamani, J.S., Miller, S.M., Spaulding, M.L. y Zimmerman, J.B., Enhanced arsenic removal using mixed metal oxide impregnated chitosan beads, Water Research, 46(14), 4427-4434 (2012) 